\title{
LUNETTES HYPERCONNECTÉES ET ALTÉRITÉ
}

Maxime Derian

\author{
C.N.R.S. Editions | « Hermès, La Revue »
}

2014/1 n 68 | pages 127 à 132

ISSN 0767-9513

ISBN 9782271080745

Article disponible en ligne à l'adresse :

https://www.cairn.info/revue-hermes-la-revue-2014-1-page-127.htm

\section{Pour citer cet article :}

Maxime Derian, «Lunettes hyperconnectées et altérité 》, Hermès, La Revue 2014/1 ( $\left.\mathrm{n}^{\circ} 68\right)$, p. 127-132.

Distribution électronique Cairn.info pour C.N.R.S. Editions.

(c) C.N.R.S. Editions. Tous droits réservés pour tous pays.

La reproduction ou représentation de cet article, notamment par photocopie, n'est autorisée que dans les limites des conditions générales d'utilisation du site ou, le cas échéant, des conditions générales de la licence souscrite par votre établissement. Toute autre reproduction ou représentation, en tout ou partie, sous quelque forme et de quelque manière que ce soit, est interdite sauf accord préalable et écrit de l'éditeur, en dehors des cas prévus par la législation en vigueur en France. Il est précisé que son stockage dans une base de données est également interdit. 


\section{Maxime Derian}

ISCC

Centre de recherches Psychanalyse,

médecine et société - université Paris 7

\section{Lunettes hyperconnectées et altérité}

Le projet Google Glass, lancé par Google, ambitionne de commercialiser massivement dans les années à venir des lunettes dotées d'un affichage head up display permettant de visionner images, vidéos et informations issues du réseau Internet, superposées, à notre convenance, à notre perception «naturelle» de l'environnement réel. Des modèles expérimentaux sont déjà testés. Ces systèmes nous incitent dès à présent à réfléchir sur l'impact de l'usage intensif de techniques de wearable computing ${ }^{1}$ sur la structuration de notre élaboration des liens sociaux, sur la socialisation et sur la question même de l'altérité.

En plus de l'image numérique - projetée sur un prisme en face d'un seul des yeux -, cette machine dispose de nombreuses autres possibilités encore en phase exploratoire. Des sons peuvent être transmis par les os du crâne (donc totalement inaudibles pour toute autre personne que l'utilisateur) ou par un microphone. De même, la machine peut enregistrer des pistes audio. Ces lunettes disposent aussi d'une caméra capable de filmer comme de prendre des photos, ainsi que d'un accéléromètre, d'un gyroscope et d'un GPS. Enfin, une interface tactile de type touchpad ou vocale permet d'interagir avec l'appareil pour filmer, téléphoner, consulter le Web. Dès leur conception, les Google Glass ont vocation à être en permanence branchées à Internet.

En corollaire, ces lunettes «hyperconnectées » représentent une potentielle multitude d'yeux, d'oreilles et de relais de diffusion audiovisuelle pour l'entreprise privée Google. Au vu de la puissance économique et commerciale qu'elle représente à présent et de sa popularité, il y a peu de risque à annoncer que ces dispositifs vont être très populaires quand ils seront accessibles pour le grand public. Depuis à peine un an, ils attisent déjà les désirs technophiles et les craintes technophobes.

De nombreux enjeux anthropologiques sont soulevés par ce probable essor de l'accès à un système de réalité augmentée couplé à une capacité d'enregistrement en vue subjective à différents capteurs de positionnement, de géolocalisation et à un accès à Internet. La diffusion des Google Glass représente finalement peut-être surtout une expérimentation sociologique et psychologique à très grande échelle.

Les situations de " présence absente ${ }^{2}$ » quel'on observe déjà quotidiennement dans certains lieux seront vraisem- 
blablement encore plus fréquentes. En effet, les lunettes de réalité augmentée, en rapprochant l'écran au plus près de l'œil, permettront à des individus de se retrouver côte à côte et pourtant dans des environnements informationnels très différents. Plus encore qu'avant, nous risquons de nous sentir «seuls à plusieurs» (Turkle, 2011). Les notions d'altérité et d'identité peuvent-elles être vraiment globalement bouleversées par un contexte d'usage massif de lunettes de réalité augmentée?

\section{La réalité augmentée ${ }^{3}$ : hybridation en temps réel des environnements physiques et numériques}

Avec les Google Glass et les modèles équivalents élaborés par différents acteurs (Massachusetts Institute of Technology, Panasonic, Epson, Sony, Oakley, Baidu, etc.), un nombre peut-être très important d'individus vivront de long laps de temps journalier dans des univers perceptifs hybrides. L'écran du smartphone, de la télévision, de la visioconférence, du jeu vidéo et du réseau social sera disponible en permanence pour le regard. Ultérieurement, des lentilles de contact prendront peut-être le relais comme système d'affichage de réalité augmentée.

Les plans, textes, images et vidéos seront affichables en temps réel sur nos rétines. La réalité médiatisée par la prothèse cognitive pourrait fortement banaliser l'incrustation d'images numériques dans notre champ visuel. Cela donnera la possibilité technique de tromper nos sens en nous procurant des sortes d'hallucinations numériques. Les actions quotidiennes peuvent alors devenir des prétextes ou des supports pour se distraire ou interagir intempestivement avec des services en ligne à vocation commerciale qui ne manqueront pas, une fois de plus, de tenter de capter notre attention et nos données personnelles. Ces outils risquent de promouvoir une «ludi- fication» de la vie quotidienne représentant une certaine forme d'infantilisation des utilisateurs, même adultes.

Par exemple, des jeux vidéo de type FPS (First Person Shooter, catégorie de jeux vidéo se déroulant en $3 \mathrm{D}$ et en vue subjective) seront vraisemblablement utilisables avec les versions améliorées des Google Glass. On verra peut-être des joueurs, dotés de pistolets invisibles, se courir après dans les rues. Ils effectueraient alors une sorte d'étrange ballet dont les tenants et les aboutissants seraient complètement hermétiques aux yeux des individus non connectés... Cela peut laisser présager la possibilité d'observer bon nombre de nouvelles situations sociales étrangement autistiques. Ingress, créé en 2012, est le premier jeu officiel de Google. Il vise à constituer des collectifs d'individus mis en réseau via une application commune en réalité augmentée sur smartphone. Une nouvelle forme de jeu vidéo encore embryonnaire va peutêtre bientôt prendre un essor conséquent: les Augmented Reality Multiplayer Online Role-Playing Game (nous proposons l'acronyme ARMORPG ${ }^{4}$ ).

Aux États-Unis, le tribunal de San Diego a récemment créé une jurisprudence favorable au port de Google Glass au volant d'une automobile. La voie est de plus en plus dégagée pour l'autorisation de porter ces lunettes hyperconnectées tout le temps et en tous lieux ${ }^{5}$. Les bénéfices économiques escomptés par la firme de Mountain View se basent principalement sur des recettes publicitaires. Ce qui prime, en termes de business model, pour ce géant de l'Internet, c'est la valorisation économique optimale de notre présence en ligne afin de pouvoir rentabiliser un maximum de nos clics de souris et de nos instants d'attention disponible. Les lunettes représentent alors un moyen de faciliter encore davantage l'accès au Web, à tout moment de la journée.

Manuel Castells (2006) a annoncé l'avènement des «médias de masse individuels». Le cours des événements semble lui donner raison. L'humanité se trouve peu à peu emmaillotée dans un filet numérique dont il devient 
extrêmement difficile de se passer. Ainsi, les élèves français passent désormais plus de temps devant un écran que devant leur professeur (Desmurget, 2011), et le citoyen français moyen passe aujourd'hui un temps considérable à interagir avec des outils numériques.

La tentation sera grande pour certaines personnes de vivre en permanence dans une forme de «vie hallucinée». Avec l'essor de la réalité augmentée, de nombreux nouveaux usages encore insoupçonnés des outils numériques vont pouvoir émerger. Une simple table pourra servir d'écran, de clavier comme de piano. Une fois équipé de lunettes performantes de réalité augmentée, il serait tout à fait possible de pouvoir voir, entre autres, un «lapin blanc» en images de synthèse traverser apparemment notre environnement immédiat. Les premiers chapitres des Aventures d'Alice au pays des merveilles pourraient ne plus sembler fantastiques aux enfants de demain, s'ils deviennent complètement habitués à la réalité augmentée dès leur berceau.

Pour Jacques Rancière (2009), l'écran est le lieu d'un «transfert construit», qui peut anticiper le regard du spectateur et donc le manipuler. L'iconoclasme byzantin du VIII ${ }^{\mathrm{e}}$ siècle considérait l'image comme «un entonnoir pour le regard» (Mondzain, 1996). C'est pour cela qu'il condamnait fermement la fascination résultant de la contemplation d'images; les iconophiles considéraient, à l'inverse, que les images qu'ils appelaient icônes étaient des moyens d'appréhender le divin et son incarnation. Cette ambivalence du rôle social des images se retrouve d'une certaine façon avec les enjeux éthiques liés à la réalité augmentée et l'informatique «seconde peau».

Comme ces lunettes pourront être portées toute la journée, d'énormes confusions pourraient être induites par une exposition sur du long terme à de telles «hallucinations» numériques persistantes en temps réel. Les personnes schizophrènes pourraient notamment être confortées dans certains de leurs délires les plus pathologiques et des utilisateurs sains pourraient peu à peu, subrepticement, être déstabilisés par un usage excessif de réalité augmentée.

Ces objets techniques sont-ils des menaces pour les pédagogies traditionnelles, des éléments perturbateurs pour les interactions entre individus? Ne sont-ils pas un nouveau type d'arme de «distraction massive» (Derian, 2013)?

Lapplication d'images subliminales ${ }^{6}$ sur des Google Glass, pour des raisons de marketing, pose certainement un véritable problème éthique. Si ces lunettes se diffusent massivement, un véritable "détournement cognitif» de l'attention pourrait être mis en œuvre par des entreprises tierces dans le cadre de publicités ciblées ou d'applications variées. Des logiciels d'«agenda contextualisé» tels que Google Now, couplés aux Google Glass, sont en mesure de fournir aux humains des sortes de «GPS pour l'esprit» qui pourraient incarner des exemples nouveaux d'humains «simplifiés» au sens proposé par Jean-Michel Besnier (2012). Par ailleurs, une question se pose quant au changement insidieux qui peut survenir dans notre relation à l'autre du fait que l'on dispose d'un accès à un stock important de données personnelles concernant une personne au moment où nous la rencontrons (par exemple en cas de reconnaissance faciale par image processing associée à un profil Facebook, un compte Twitter ou LinkedIn). Après tout pourquoi chercher à parler à l'autre si on en sait déjà «trop» à son propos?

\section{Le statut de «l'autre»}

De manière schématique et probablement réductrice, en premier lieu, l'« autre» désigne ce qui «n'est pas nous». Ce qui parait évident d'un premier abord devient vite complexe à définir tant notre porosité corporelle et psychosensorielle est forte, qu'on le veuille ou non, à l'égard de notre milieu et des autres êtres vivants. C'est le développement psychologique de l'enfant qui lui apprend à faire 
la distinction du soi et du non-soi. La dimension symbolique des représentations et du langage est un artifice pour tenter de résoudre la difficulté de penser la communication avec «ce qui n'est pas nous».

La définition de l'altérité participe en fait, de façon essentielle, à permettre de se construire et de se penser en tant qu'individu. Il semble donc probable que ce qui peut modifier notre conception de l'altérité amène en retour à modifier notre propre identité. La négation totale de l'altérité chez un individu relève de la manifestation de la psychose ou de la perversion.

Les probables versions ultérieures des Google Glass disposeront peut-être d'une capacité de calcul suffisante pour pouvoir déformer ou soumettre à des effets spéciaux, en temps réel, les images que nous voyons naturellement. La voie sera alors libre pour que certains murs blancs du monde réel puissent servir à l'affichage de publicités contextualisées numériques ${ }^{7}$, et il sera techniquement faisable de faire apparaître des créatures imaginaires dans notre champ visuel ${ }^{8}$. Des «applications» Android pourraient être en mesure de transformer à notre grél'apparence d'un interlocuteur en co-présence physique au moyen d'un avatar numérique surimposé sur l'image réelle de la personne. Il serait alors possible, pour un porteur de lunettes de réalité augmentée, de se tenir devant une personne réelle ayant à ses yeux l'apparence de Marylin Monroe ou de Bugs Bunny, par exemple.

Or, pour l'utilisateur de ces dispositifs, «l'autre» des lunettes hyperconnectées ne sera pas seulement un autre être humain. Même si les machines ne réussissent pas encore à passer le test de Turing avec succès, il semble que notre fréquentation assidue des prothèses cognitives tende à nous habituer à interagir avec différentes formes «d'autres» qui tendent à se confondre, puisqu'ils sont «rencontrés» sur un même support. Jaron Lanier (2010) considère que des interactions répétées avec un outillage informatisé non implanté peuvent vraisemblablement provoquer une certaine identification entre l'utilisateur et l'automate qu'il actionne. Il observe un processus de standardisation des modes de présentation et de représentation de soi, qui serait généré, selon lui, par un usage excessif de programmes au cours de la vie quotidienne.

Les lunettes de réalité augmentée permettent de mélanger dans notre champ visuel plusieurs «interlocuteurs» de natures différentes. L'autre peut être soit un individu en chair et en os en situation de co-présence dans un même lieu, soit l'avatar d'un individu situé dans un endroit distant, soit un programme, un bot accessible via l'interface et représenté par un avatar lui aussi. L'inédit de la situation résulte d'une possible confusion entre les trois «altérités» en raison d'une exposition très prolongée à des informations numériques en mesure de tromper les sens des utilisateurs.

Les Google Glass actuelles rendent déjà possible des situations assez atypiques d'interactions sociales. Elles peuvent ainsi être utilisées pour retransmettre une conversation vidéo en temps réel à des spectateurs distants en mesure de parler en toute discrétion (via les micros à transmission par os) avec l'utilisateur. En s'adressant à un porteur de Google Glass, une personne peut croire parler à un seul individu alors qu'elle s'adresse en réalité à tout un groupe.

Les «lunettes hyperconnectées» ne sont pas seulement une rupture technologique permettant de rendre des réalités augmentées accessibles à tout instant. Comme ces machines sont des dispositifs prévus pour être constamment branchés sur Internet, elles diffuseront des informations issues du réseau à leur porteur et, d'autre part, elles propageront des données intimes concernant l'utilisateur et l'environnement de ce dernier. Ces outils, mis à disposition de tous pourraient accentuer davantage l'engouement actuel pour la tendance au quantified self et au lifelogging 9 .

Le port de ces lunettes implique donc, dans une certaine mesure, l'acceptation tacite par l'usager d'une forme de négation du droit des «autres» à la vie privée. En effet, alors que le porteur pourra régler ses propres paramètres 
de sécurité, la personne filmée à son insu (dans un lieu public comme privé) pourra être reconnue, identifiée et localisée par les logiciels installés sur les serveurs de l'entreprise Google. Dans un tel cas de figure, nier le droit à la vie privée de l'autre participe à avaliser l'idée très orientée (par Facebook, Google et consorts) que la vie privée serait devenue obsolète. Accepter de nier le droit à l'intimité de l'autre aura pour effet, à plus ou moins brève échéance d'abandonner son propre droit à l'intimité. Par ricochet, le port généralisé de lunettes connectées pourrait finalement contribuer à considérablement accroître la société de surveillance généralisée (étatique ou non).

Avec la fonctionnalité d'enregistrement en vue subjective, il sera aisé de capturer des instants du quotidien afin de les «revivre» ultérieurement. Sur ce point, Saadi Lahlou (qui a mené des expériences de ce type avec la Subcam) évoque le fait qu'avoir accès à des enregistrements en vue subjective de nos interactions sociales peut provoquer certains chocs psychologiques. Cette extranéation hégélienne, cette possibilité de prise de recul, mise à disposition électroniquement, semble en mesure de perturber notre «rapport à l'autre». Se revoir en interaction avec quelqu'un peut complètement modifier un ressenti initial.
L'enregistrement en vue subjective permet de "voir avec les yeux d'un autre». Cela aussi peut s'avérer déstabilisant pour les relations sociales. De nombreuses dérives notamment illustrées par le film Strange Days de Kathryn Bigelow (1995) - peuvent découler de ce type de pratiques facilitées encore une fois par les lunettes de Google.

Les prothèses cognitives ne nous permettent donc pas nécessairement de «mieux» communiquer; au contraire, parfois, elles nous isolent davantage les uns des autres, voire sont utilisées à notre insu pour faciliter le contrôle des autorités étatiques et privées sur nos comportements individuels et collectifs ${ }^{10}$, ou pour faire fructifier un capital économique. Jaron Lanier (2013) nomme «serveurs siréniques» ces dispositifs de récolte massive d'information alimentés bénévolement par les usagers et qui donnent de la valeur ajoutée à un logiciel contrôlé par un nombre réduit d'informaticiens (Twitter, Snapchat, etc.).

L'utilisation d'une prothèse cognitive peut servir à entrer en contact avec de nombreuses autres personnes. La plupart du temps, cependant, l'usager est, au final, essentiellement confronté à des programmes informatiques.

\section{NOTES}

1. Le wearable computing s'inscrit dans le domaine de l'informatique "pervasive», néologisme pour décrire une tendance technique pénétrante et ubiquitairement invasive de l'informatique. Cf. Weiser, 1991.

2. La présence absente est un concept que nous proposons pour dépeindre le comportement d'un humain physiquement parmi un groupe d'individus mais dont l'attention est ailleurs, captée par un mécanisme électronique. Cette notion complète l'idée de présence concrète telle qu'on la retrouve chez Anders (2002). Sherry Turkle (2011) utilise les termes "Alone Together», que l'on peut traduire par «solitude à plusieurs».

3. Selon Steve Mann (2002), le concept de «réalite virtuelle» fut initialement proposé par Jaron Lanier en 1989 et l'expression «réalité augmentée» fut inventée par Tom Caudelle au début des années 1990.

4. Ces jeux n'auront pas comme caractéristique essentielle d'être massivement multi-utilisateurs, mais de se dérouler en réalité augmentée.

5. Certains lieux privés et publics ont déjà opté pour l'interdiction du port de Google Glass. Mais il est vraisemblable que les effets de telles interdictions demeureront limités en cas de déferlement des outils... 
6. L'utilisation d'images subliminales en réalité augmentée est expérimentée au Massachusetts Institute of Technology, avec "The MIT Memory Glasses Project". En ligne sur: <www. media.mit.edu/wearables/mithril/memory-glasses.html $>$., consulté le 31/01/2014.

7. Les protagonistes du récit The Golden Age (Wright, 2002) sont littéralement assaillis par les publicités en réalité augmentée dans leur vie de tous les jours.

8. Dans la série télévisée Battlestar Galactica (de Ronald D. Moore, 2003), les humanoïdes synthétiques (Cylons) vivent dans un environnement minimaliste qu'ils agrémentent d'une décoration personnalisée en réalité augmentée.

\section{RÉ F ÉRENCES B I B L IOGR A P H I Q U E S}

Anders, G., L'Obsolescence de l'homme, t. 1, Paris, Encyclopédie des nuisances, 2002.

BESNIER, J.-M., L'Homme simplifié. Le syndrome de la touche étoile, Paris, Fayard, 2012.

CAstells, M., "Émergence des "médias de masse individuels" ", Le Monde diplomatique, août 2006.

Derian, M., Le Métal et la chair. Anthropologie des prothèses informatisées, thèse de doctorat en sociologie, Paris, université Paris 1 Panthéon Sorbonne, 2013.

Desmurget, M., TV Lobotomie. La vérité scientifique sur les effets de la télévision, Paris, Max Milo, 2011.

LANIER, J., You Are Not a Gadget, New York, Knopf, 2010.

LANiER, J., Who Owns the Future, New York, Simon \& Schuster, 2013.
9. Le lifelogging (autrement nommé le quantified self) consiste à procéder à des enregistrements systématisés d'un maximum de données concernant notre vie. Il peut s'agir d'enregistrements vidéo ou audio, de scans de documents, d'historiques de consultation du Web, d'historiques de comptes bancaires, d'enregistrement de localisation GPS, mais aussi de la collecte de différents paramètres physiologiques (poids, température, pression artérielles, rythme cardiaque, etc.).

10. Les récentes révélations d'Edward Snowden à propos du système généralisé d'espionnage mondial de la part de la National Security Agency confortent la plupart des soupçons à ce sujet.

MANN, S., «Mediated Reality With Implementations for Everyday Life», Presenceconnect.com [en ligne], 6 août 2002.

Mondzain, M.-J., Image, icône, économie, Les sources byzantines de l'imaginaire, Paris, Seuil, 1996.

RANCIÈRE, J., Et tant pis pour les gens fatigués: entretiens, Paris, éditions Amsterdam, 2009.

Turkle, S., Alone Together. Why We Expect More from Technology and Less from Each Other, New York, Basic Book, 2011.

Weiser, M., "The Computer for the 21st Century», Scientific American, vol. 265, n³, 1991, p. 94-104.

Wright, J. C., The Golden Age, vol. 1, New York, MacMillan, 2002. 